\title{
Quasi-Conformally Flat Mapping the Human Cerebellum
}

\author{
Monica K. Hurdal ${ }^{1}$, Philip L. Bowers ${ }^{1}$, Ken Stephenson ${ }^{2}$, \\ De Witt L. Sumners ${ }^{1}$, Kelly Rehm ${ }^{3,4}$, Kirt Schaper $^{3}$, and \\ David A. Rottenberg ${ }^{3,4}$
}

1 Dept. of Mathematics, Florida State University, Tallahassee, FL 32306-4510, USA mhurdal@math.fsu.edu

http: //www.math.fsu.edu/ ${ }^{\sim}$ mhurdal

2 Dept. of Mathematics, University of Tennessee, Knoxville, TN 37996-1300, USA

3 PET Imaging Center, VA Medical Center, Minneapolis, MN, 55417, USA

4 Dept. of Radiology, University of Minnesota, Minneapolis, MN, 55455, USA

\begin{abstract}
We present a novel approach to creating flat maps of the brain. It is impossible to flatten a curved surface in 3D space without metric and areal distortion; however, the Riemann Mapping Theorem implies that it is theoretically possible to preserve conformal (angular) information under flattening. Our approach attempts to preserve the conformal structure between the original cortical surface in 3-space and the flattened surface. We demonstrate this with data from the human cerebellum and we produce maps in the conventional Euclidean plane, as well as in the hyperbolic plane and on a sphere. Conformal mappings are uniquely determined once certain normalizations have been chosen, and this allows one to impose a coordinate system on the surface when flattening in the hyperbolic or spherical setting. Unlike existing methods, our approach does not require that cuts be introduced in the original surface. In addition, hyperbolic and spherical maps allow the map focus to be transformed interactively to correspond to any anatomical landmark.
\end{abstract}

\section{Introduction}

The human brain can be divided into regions based on function and anatomy [16]. These divisions are generally determined by the location of folds (gyri) and fissures (sulci) in the cortical surface. However, the surface of the brain is extremely convoluted, with considerable anatomical variability between individuals. The combination of fold complexity with anatomical variability makes it difficult to compare anatomical and functional information within and between subjects. Current visualization and comparison techniques do not satisfactorily overcome these difficulties. For example, functional (PET, fMRI, etc.) data can be projected onto a rendering of the cortical surface extracted from a coregistered magnetic resonance $(\mathrm{MR})$ volume. Individual differences in cortical folding make it difficult to compare the location and extent of activated foci. Foci buried in deep sulci may appear on the cortical surface and widely separated foci on opposite walls of a sulcus may appear to be close together. 
Interestingly, the surface representing the cortical grey matter is topologically equivalent to a two-dimensional sheet. Thus, it is possible to unfold or flatten this surface to create a $2 \mathrm{D}$ flat map of the cortex. This surface-based approach can assist in visualizing and comparing cortical folding patterns and help to resolve some of the problems which exist in traditional visualization techniques.

A number of computational tools have been developed to take advantage of this surface-based approach. Drury et al. [7] have developed an approach that attempts to reduce the areal and angular distortion between the original cortical surface in $3 \mathrm{D}$ space and the flattened surface in $2 \mathrm{D}$ space. This is accomplished by iteratively applying torsional and longitudinal forces to adjust the edge lengths in the mesh. Fischl et al. [8] create a flat map by reducing geodesic distance distortion while attempting to prevent folds in the flattened surface. Both techniques have been used successfully in several comparative and functional investigation studies $[5,6,13,14,15]$.

In this paper we present a novel flat mapping approach that attempts to preserve the conformal structure between the original cortical surface and the flattened surface. There are two real advantages to using conformal flattening as opposed to others. First, it is completely canonical, meaning that such flattenings are uniquely determined once certain standard normalizations have been chosen. In particular, it requires no ad hoc cutting of the surface as do some of the previously described methods for surface flattening [7,8]. Second, the flattening can be done in either the Euclidean plane or in the hyperbolic plane, and the surface can also be mapped conformally onto a sphere. Though the setting of the hyperbolic plane might seem merely incidental and of little practical value, its use allows the map origin to be transformed interactively so that different anatomical landmarks can be used as the map focus. This has the advantage of bringing into sharp relief areas of interest, but at the cost of relegating the region far removed from the origin to the periphery, where our Euclidean eyes cannot focus. The primary disadvantage of conformal flattening is that there is no attempt to control either the metric or areal distortion; indeed, there can be no such control as the conformal flattening is canonical and offers no such opportunity without the loss of conformality.

\section{Quasi-Conformal Flattening}

In constructing flat mappings of anatomical surfaces such as the cerebellum, one is faced with two major problems. The first is that one must take the raw 3D scan data and use it to produce a mesh of the 2D surface of interest. Computational triangulation methods usually result in a topologically complicated surface replete with local artifacts caused by several factors, including the peculiarities of certain standard algorithms and software packages (e.g. the marching cubes algorithm) and insufficient spatial resolution of the data. These local artifacts must be removed by slicing, patching and retriangulating the surface until a simply connected 2D mesh is obtained in 3D space that conforms to the surface of interest. In this section, we are concerned with the second major problem 
- that of flattening the highly convoluted mesh that conforms to the original surface once it has been converted into a topologically defect-free piecewise flat surface in 3D space. There is no way to flatten a curved 3D surface without metric and areal distortion [11]; however, there is one type of geometric information that, at least theoretically, can be preserved under flattening, namely conformal (angular) information encoded in the surface.

Our starting point is a piecewise flat triangulated surface in $3 \mathrm{D}$ space that is topologically a $2 \mathrm{D}$ disk. The $3 \mathrm{D}$ space coordinates of the vertices of each triangle of the mesh are given, and each edge of the mesh is either an interior edge contained in exactly two triangles, or a boundary edge contained in exactly one triangle. There is one boundary component - a single closed chain of boundary edges forming the boundary of the surface. Technically, this piecewise flat surface carries the structure of a Riemann surface (described below), and by the Riemann Mapping Theorem [1] it conformally maps onto any proper simply connected region in the complex plane. Our job is to describe a tool for producing (approximations to) these conformal mappings.

\subsection{Riemann Surfaces}

The technical description of a Riemann surface is in terms of a complex atlas: a collection of local mappings that provide local complex coordinates on the surface so that overlap mappings are analytic [2]. Rather than going into a detailed technical description for the uninitiated reader, it serves our purposes to understand that a Riemann surface provides a consistent way to measure angles that gives a full angle measure of $2 \pi$ around each interior point, including interior vertices. For piecewise flat surfaces where all but the finitely many vertices have small neighborhoods isometric to disks in the Euclidean plane, the measure of an angle based at a point other than one of the triangle vertices is just the Euclidean measure of that angle. For an angle based at a vertex the measure determined by the complex atlas of the Riemann surface is the Euclidean measure linearly rescaled so that the total angle measure is $2 \pi$. Explicitly, denote by $\Theta(v)$ the angle sum about the vertex $v$, i.e. the sum of the Euclidean measures of the angles with vertex $v$ from all of the triangles of the triangulation that contain $v$. The scale factor used at $v$ for measuring angles is then $\frac{2 \pi}{\Theta(v)}$. Hence, an angle with vertex $v$ in our surface that has Euclidean measure $\theta$ will be found to have measure $\frac{2 \pi \theta}{\Theta(v)}$ in the complex atlas of the Riemann surface. A conformal mapping of a Riemann surface to another one is a continuous locally one-to-one function that preserves all angle measures. Hence a conformal mapping from our piecewise flat surface to the complex plane is one that preserves the Euclidean angle measures at non-vertices, and maps neighborhoods of the vertices in such a way that the "market share" of angles is preserved, i.e. an angle based at vertex $v$ of Euclidean measure $\theta$ has image an angle of Euclidean measure $\frac{2 \pi \theta}{\Theta(v)}$ under any conformal mapping to the plane. 


\subsection{Approximating Conformal Mappings Using Circle Packings}

Our scheme for approximating conformal mappings of a piecewise flat surface to flat maps in the plane uses circle packings. Given a triangulation $K$ of a disk, a circle packing for $K$ is a collection $\mathcal{C}_{K}=\{C(v): v$ is a vertex of $K\}$ of circles in the plane, one circle for each vertex $v$ of $K$, with the property that circles $C(v)$ and $C(w)$ are tangent whenever the vertices $v$ and $w$ form an edge of $K$. The Circle Packing Theorem of [4] states that given any disk triangulation $K$ and any assignment of positive numbers $r\left(v_{1}\right), \cdots, r\left(v_{n}\right)$ to the $n$ boundary vertices $v_{1}, \cdots, v_{n}$ of $K$, there is a unique (up to Euclidean isometry) circle packing in the plane with boundary circle $C\left(v_{i}\right)$ having radius $r\left(v_{i}\right)$, for $i=1, \cdots, n$.

Our first attempt to approximate conformal mappings uses these circle packings in the following way. Starting with a piecewise flat surface in 3D space, collect the combinatorial data from the surface in an abstract triangulation $K$. This is merely a list of the vertices of the surface (not their 3-space coordinates), the pairs of vertices in edges, and the (oriented) triples of vertices in triangular faces. Assign positive numbers to the boundary vertices (perhaps set all equal to one, or to the average of the lengths of the two edges containing a given vertex) and find the unique circle packing $\mathcal{C}_{K}$ guaranteed by the Circle Packing Theorem. The packing $\mathcal{C}_{K}$ serves the role of our flat mapping of the surface and the question is how well this circle packing mapping approximates a conformal mapping. We certainly do not expect the mapping to be conformal since none of the conformal data of the piecewise flat surface are used in constructing the circle packing - it is only the combinatorial data along with arbitrary boundary radii assignments that are used in constructing the circle packing. Nonetheless, the Ring Lemma of [12] applies to guarantee that this circle packing mapping is quasi-conformal, meaning that there is but a bounded amount of percentage angular distortion. To obtain closer approximations to the actual conformal mappings requires certain modifications of the circle packing routine that are designed to reduce the quasi-conformal distortion. Unfortunately, space considerations prevent a description of the necessary modifications and details will appear elsewhere.

\subsection{Flat Maps in the Hyperbolic Plane}

This approximation scheme works just as well in the hyperbolic plane as in the Euclidean plane (i.e. the complex plane $\mathbf{C}$ ). There is a version of the Circle Packing Theorem for the hyperbolic plane where hyperbolic radii are used as well as hyperbolic lines and triangles [4]. The Poincaré disk model of the hyperbolic plane [3] consists of the unit disk $\Delta=\{z \in \mathbf{C}:|z|<1\}$ with metric determined by the line element $d s=\frac{2|d z|}{1-|z|^{2}}$, the Euclidean line element $|d z|=\left(d x^{2}+d y^{2}\right)^{1 / 2}$ scaled by twice the reciprocal of $1-|z|^{2}$. This means that the hyperbolic angle measures are the same as the Euclidean angle measures, but the hyperbolic distances are distorted when seen through our Euclidean eyes by constant hyperbolic lengths appearing smaller and smaller as the segment moves toward the 
unit circle boundary of $\Delta$. We may apply the circle packing routine described previously, using hyperbolic measurements rather than Euclidean, and obtain quasi-conformal mappings of our piecewise flat surfaces to the hyperbolic plane. The portions of the flat mapping lying near the boundary unit circle are greatly distorted to our Euclidean eyes (though not so distorted to hyperbolic eyes), but the portions lying near the origin have small distortion in comparison. The nice feature of this is that one may arbitrarily choose what vertex of our surface should be mapped to the origin. This way, different portions of the original surface can be brought into focus with more distant portions relegated to the edges, and this can be automated easily to provide an interactive tool for viewing local features of interest on our surface. Extensions that allow interactive quasi-conformal spherical mappings are also available.

\section{$3 \quad$ Flattening the Cerebellum}

We obtained a high resolution MRI scan from the Montreal Neurological Institute, which was produced by registering and intensity averaging a series of $27 \mathrm{~T} 1$ weighted images from a single subject [9]. This volume is composed of isovoxels and has dimension $217^{3}$. We chose to flatten the cerebellum in an effort to facilitate the description of activated cerebellar foci in functional neuroimaging.

The cerebellum was extracted from the MRI volume by defining a plane parallel to the posterior commissure-obex line and orthogonal to a plane passing through the vermal midline. Cortical regions defined by various lobes and fissures were color coded for identification purposes. We produced a triangulated surface representing the cerebellar surface using the marching cubes algorithm [10]. The output of this surface was checked for any topological errors and corrected as necessary. This triangulated mesh is topologically equivalent to a sphere as all edges in the surface occur exactly twice. This surface is composed of 41804 triangles with 20904 vertices and 62706 edges and is illustrated in Fig. 1. We introduced a single closed chain of boundary edges in the surface based around the brainstem and walls and apex of the fourth ventricle to act as the boundary of the flattened map. This mesh, representing the surface of the cerebellum, is a piecewise flat simply connected surface that is topologically equivalent to a disk.

Our quasi-conformal flattening procedure was applied to this surface to produce a number of flat maps. Firstly, in Fig. 2 we present the flat map that was created in the Euclidean plane. In this case, the circles for the boundary vertices were assigned a radius which was the average of the lengths of the two boundary edges containing that vertex. As with flat maps produced by other researchers $[7,8]$, the shape of this map is largely determined by the length and number of edges in the chosen boundary and will vary in shape from map to map.

Fig. 2 also illustrates a spherical mapping, where two arbitrary points may be chosen as the north and south poles and no cuts need be introduced. This representation may be more natural for some anatomical shapes. 
Flat maps that were created in the hyperbolic plane are illustrated in Fig. 3. There are a number of important features to note about these maps. Since we look at these maps with Euclidean eyes, portions of the map lying near the origin have small distortion and appear similar to those on a Euclidean map. However, regions of the map lying near the boundary of the unit circle (map border) are greatly distorted. Nevertheless, we are able to move the focus of the hyperbolic map to change the regions which are in focus and which are relegated to the map edges (with large distortion). This is the difference between the hyperbolic maps in Fig. 3. These transformations can be performed in real computation time by simply indicating the new map origin. Another feature of the hyperbolic maps is the boundary shape corresponds to the unit circle. This facilitates comparison of different maps. A coordinate system can easily be imposed by specifying two points, or a point and a direction. We do not need to introduce multiple cuts in the surface (other than the boundary); and perhaps most importantly, the maps are mathematically unique (up to certain transformations). The utility of quasiconformal maps for studying functional imaging data is yet to be determined, but we believe these features will produce favorable results.

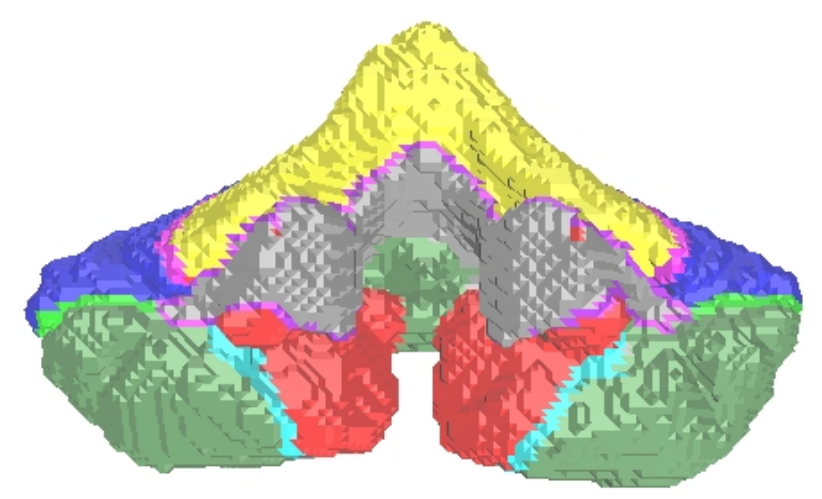

Fig. 1. Surface Representing the Cerebellum. Colors correspond to the following cortical regions: forest green = lobulus semilunaris, lobulus semilunaris inferior, lobulus biventer; red = tonsils, flocculus; yellow = lingula, lobus centralis, lobulus quadrangularis; blue = lobulus simplex, lobulus semilunaris superior; grey = white matter; bright green = fissura prima; cyan = fissura secunda; magenta $=$ fissura horizontalis; purple $=$ boundary used for flat maps.

\section{Discussion}

We have introduced a novel approach to unfolding and flattening the cortical surface which attempts to preserve the conformal structure of the original surface in 3-space. Our approach offers a number of advantages over existing approaches including: 

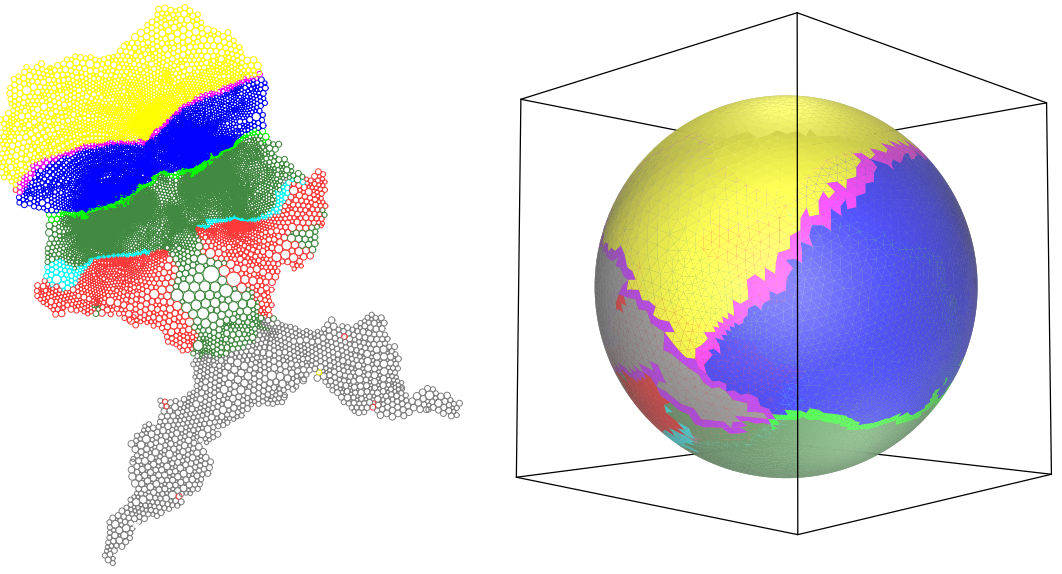

Fig. 2. Euclidean Flat Map and Spherical Map of the Cerebellar Surface. Colors correspond to regions shown in Fig. 1. A boundary is required to create a flap map of the cerebellum (left figure); however, the cerebellar surface is topologically equivalent to a sphere, so no boundary is required to quasi-conformally map it to the surface of sphere.
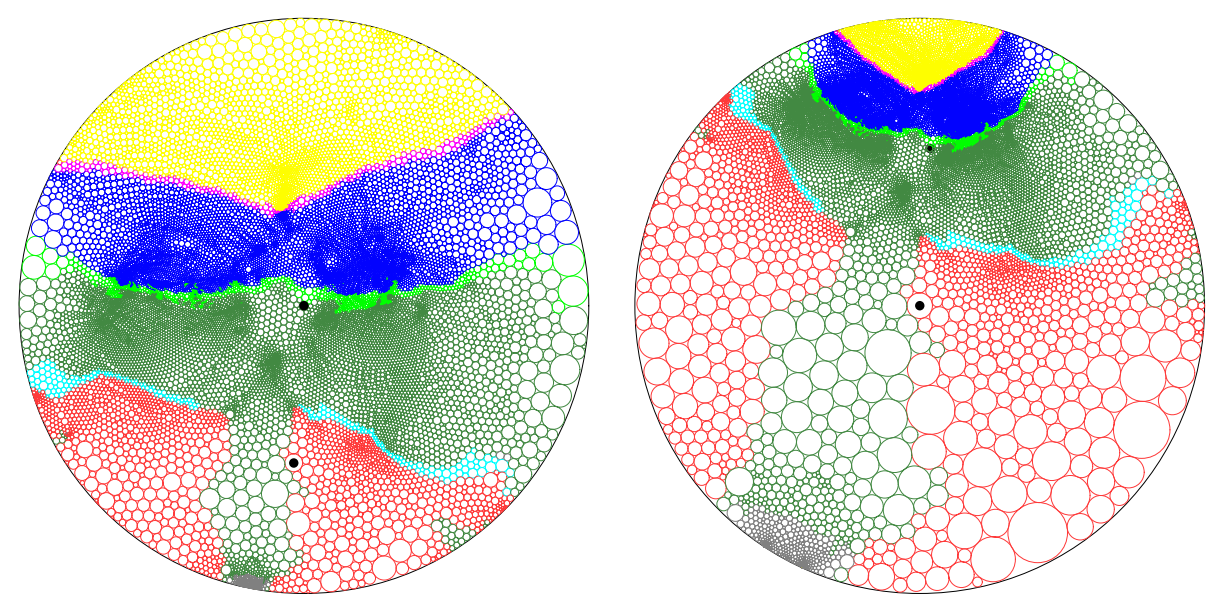

Fig. 3. Hyperbolic Flat Maps of the Cerebellar Surface. Colors correspond to regions shown in Fig. 1. The origin (map focus) is marked in black in the center of the maps. The map on the right has been transformed to a different map focus to display regions in the lower portion of the map. The black circle located near the bottom of the left map is used as the map origin in the right map. Similarly, the black circle located near the top of the right map corresponds to the map focus of the left map. 
- conformal mappings control and minimize angular distortion

- conformal mappings are canonical, and hence mathematically unique

- cutting of the original surface is not required to reduce distortion

- flattening can be done in the Euclidean and hyperbolic planes and mapping to a sphere is also possible

- a coordinate system can be easily imposed on the hyperbolic maps

- the map origin in the hyperbolic plane can be transformed interactively to change the map focus and alter the primary locations of map distortion

- computation times are on the order of minutes with real time user interaction.

Our approach produces quasi-conformal maps which may allow us to better localize functional regions of activation in normal subjects and patients with ataxia and other hereditary diseases. We are currently working on extensions that will produce closer approximations to the true conformal mapping.

This work is supported in part by NIH grants MH57180 and NS33718.

\section{References}

1. Ahlfors, L. V.: Complex Analysis. McGraw-Hill Book Company, New York, 1966 281

2. Beardon, A. F.: A Primer on Riemann Surfaces. Cambridge University Press, Cambridge, 1984, LMS Lecture Notes 78281

3. Beardon, A. F.: An introduction to hyperbolic geometry, in Ergodic Theory, Symbolic Dynamics, and Hyperbolic Spaces, T. Bedford, M. Keane, and C. Series, eds., Oxford University Press, Oxford, 1991, ch. 11, pp. 1-34 282

4. Beardon, A. F., Stephenson K.: The uniformization theorem for circle packings. Indiana Univ. Math. J. 39 (1990) 1383-1425 282

5. Corbetta, M.: Frontoparietal cortical networks for directing attention and the eye to visual locations: Identical, independent, or overlapping neural systems? Proc. Natl. Acad. Sci. USA. 95 (1998) 831-838 280

6. Drury, H .A., Van Essen, D. C.: Functional specializations in human cerebral cortex analyzed using the visible man surface-based atlas. Hum. Brain Mapping 5 (1997) 233-237 280

7. Drury, H .A., Van Essen, D. C., Anderson, C. H., Lee, C. W., Coogan, T. A., Lewis, J. W.: Computerized mappings of the cerebral cortex: A multiresolution flattening method and a surface-based coordinate system. J. Cog. Neurosci. 8 (1996) 1-28 280, 283

8. Fischl, B., Sereno, M. I., Dale, A. M.: Cortical surface-based analysis II: Inflation, flattening, and a surface-based coordinate system. Neuroimage 9 (1999) 179-194 280,283

9. Holmes, C. J., Hoge, R., Collins, L., Evans, A. C.: Enhancement of T1 MR images using registration for signal averaging. J. Neurosci. 3 (1996) S28, Part 2 of 2283

10. Lorensen, W. E., Cline, H.: Marching cubes: A high resolution 3D surface construction algorithm. Computer Graphics 21 (1987) 163-169 283

11. Polya, G.: Mathematical Discovery, vol. 2. John Wiley \& Sons, New York, 1968 281

12. Rodin, B., Sullivan, D.: The convergence of circle packings to the Riemann mapping. J. Differential Geometry 26 (1987) 349-360 282 
13. Tootell, R. B. H., Hadjikhani, N. K., Vanduffel, W., Liu, A. K., Mendola, J. D., Sereno, M. I., Dale, A. M.: Functional analysis of primary visual cortex (V1) in humans. Proc. Natl. Acad. Sci. USA. 95 (1998) 811-817 280

14. Tootell, R. B. H., Mendola, J. D., Hadjikhani, N. K., Liu, A. K., Dale, A. M.: The representation of the ipsilateral visual field in human cerebral cortex. Proc. Natl. Acad. Sci. USA. 95 (1998) 818-824 280

15. Van Essen, D. C., Drury, H. A., Joshi, S., Miller, M. I.: Functional and structural mapping of human cerebral cortex: Solutions are in the surfaces. Proc. Natl. Acad. Sci. USA. 95 (1998) 788-795 280

16. Zeki, S.: A Vision of the Brain. Blackwell Scientific Publications, Oxford, 1993279 